EPJ Web of Conferences 13, 02001 (2011)

DOI: $10.1051 /$ epjconf/20111302001

C Owned by the authors, published by EDP Sciences, 2011

\title{
Facets of the QCD Phase-Diagram
}

\author{
Volker Koch ${ }^{1, \mathrm{a}}$, Adam Bzdak ${ }^{1, \mathrm{~b}}$, and Jinfeng Liao ${ }^{2, \mathrm{c}}$ \\ 1 Lawrence Berkeley National Laboratory \\ Berkeley, CA 94720, USA \\ 2 Brookhaven National Laboratory \\ Upton, NY 11796, USA
}

\begin{abstract}
In this contribution we will discuss two aspects of the matter created in ultra-relativistic heavy ion collisions. First we will attempt to define a universal measure for the fluidity of a substance, which will allow a correct comparison between the fluidity of a Quark Gluon Plasma and any well known substance. Second we will discuss current measurements of particle correlations and their implication for possible local parity violation.
\end{abstract}

\section{Introduction}

Experiments at the Relativistic Heavy Ion Collider (RHIC) have revealed quite a number of interesting and surprising results. Among them is the rather strong elliptic flow [1], which seems to suggest almost ideal fluid-dynamic expansion of the system created in these collisions (see e.g [2,3] for a recent review). This observation has lead to the conjecture that the matter created in these collisions is strongly interacting, with nearly ideal fluidity and the phrase "perfect fluid" has been coined to describe the matter at RHIC. This was mostly inspired by the observation that a large class of gauge theories exhibit a lower bound on the ratio of shear viscosity over entropy-density, $\eta / s \geq 1 /(4 \pi)$, in the limit of very large coupling [4]. And indeed it seems that the ratio of $\eta / s \geq 1 /(4 \pi)$ appears to be a lower limit to all known substances $[5,6]$ including quantum liquids and cold Fermi gases [7]. However, it is not obvious to which extent the ratio $\eta / s$ controls or defines the fluidity of a substance. It certainly does not enter naturally in the non-relativistic Navier-Stokes equation. To clarify this situation, in the first part of this contribution we will discuss how one can define a measure of fluidity which is equally applicable for both relativistic fluids, such as the Quark Gluon Plasma (QGP) as well as non-relativistic fluids, such as water. Details can be found in [8].

Another potentially interesting aspect of heavy ion collisions is that they may be utilized to detect local parity violation due to the non-trivial topological properties of the strong interaction, QCD. The suggestions by Kharzeev and collaborators that the topological sphaleron transitions together with a chirally restored phase created in heavy ion collisions could result in the so-called Chiral Magnetic Effect (CME) [9]. The Chiral Magnetic Effect predicts that in

\footnotetext{
a e-mail: vkoch@lbl.gov

b e-mail: ABzdak@lbl.gov

c e-mail: JLiao@bnl.gov
}

the presence of the strong external (electrodynamic) magnetic field at the early stage after a (non-central) collision sphaleron transitions induce a separation of charges along the direction of the magnetic field. Of course, sphaleron and anti-sphaleron transitions are equally likely, and, therefore, the event-averaged charge separation will vanish. However, if present, the event-by-event charge separation should be observable in a suitable correlation measurement. Such a measurement has been proposed by Voloshin in [10] and recently carried out by the STAR collaboration [11,12]. Here we will discuss to which extend the STAR measurement is indeed sensitive to the CME. Details can be found in [13-15]

\section{A universal measure for fluidity}

Let us start this section by reminding ourselves about the non-relativistic Navier-Stokes equation [16] (in the absence of bulk viscosity)

$$
\left[\partial_{t}+\mathbf{v} \cdot \nabla\right] \mathbf{v}=-\frac{\nabla p}{\rho}+\frac{\eta}{\rho} \nabla_{\mathbf{j}} \Sigma^{\mathbf{j i}}
$$

with the non-relativistic shear tensor $\Sigma_{j i}=\partial_{j} v_{i}+\partial_{i} v_{j}-$ $\frac{2}{3} \delta_{j i} \nabla \cdot \mathbf{v}$. From this expression, one already sees that the dissipative term is controlled by the ratio of the shear viscosity $\eta$ over the mass-density $\rho$, commonly referred to as the kinematic viscosity [16]

$$
v \equiv \frac{\eta}{\rho}
$$

This is similar to classical mechanics where the ratio of friction over inertia and not the friction by itself controls the dynamics. And indeed, while the shear viscosity of water is a hundred times larger than that of air, its kinematic viscosity is a factor fifteen smaller, supporting our

This is an Open Access article distributed under the terms of the Creative Commons Attribution-Noncommercial License 3.0, which permits unrestricted use, distribution, and reproduction in any noncommercial medium, provided the original work is properly cited. 
everyday notion that water is a better fluid than air. We further note the absence of a term containing the widely discussed ratio $\eta / s$ in the non-relativistic Navier-Stokes equation. Hence it is rather unlikely that this ratio can serve a measure for the fluidity of non-relativistic fluids, such as water. Considering the corresponding relativistic NavierStokes equation [16],

$$
\begin{aligned}
\gamma^{2}\left[\partial_{t}+\mathbf{v} \cdot \nabla\right] \mathbf{v}= & -\frac{1}{w / c^{2}}\left[\nabla p+\frac{\mathbf{v}}{c} \partial_{0} p\right] \\
& +\frac{\eta}{w / c^{2}} \partial_{v} \boldsymbol{\Sigma}^{v \mathbf{i}}
\end{aligned}
$$

with the relativistic shear tensor $\Sigma_{\mu \nu}=c\left[\partial_{\mu} u_{v}+\partial_{\nu} u_{\mu}-\right.$ $\left.(u \cdot \partial) u_{\mu} u_{v}+\frac{2}{3}\left(u_{\mu} u_{v}-g_{\mu v}\right)(\partial \cdot u)\right], \gamma=1 / \sqrt{1-v^{2} / c^{2}}, u_{\mu}=$ $\gamma(1, \mathbf{v} / c)$, and $\partial_{0}=\frac{1}{c} \partial_{t}$. The essential difference from the non-relativistic version is that the inertia is now given by the enthalpy-density $w$ instead of the mass-density $\rho$. Thus the generalized form for the kinematic viscosity is

$$
v=\frac{\eta}{w}
$$

The enthalpy-density is given by

$$
w=\epsilon+p=T s+\mu n
$$

where $n$ is the particle density. In the non-relativistic limit, $T \ll \mu$ so that $\mu \rightarrow m$ and

$$
\begin{aligned}
& w \stackrel{T \ll \mu}{\longrightarrow} m n=\rho, \\
& v \stackrel{T \ll \mu}{\longrightarrow} \frac{\eta}{\rho},
\end{aligned}
$$

recovering the non-relativistic Navier-Stokes equation. In the relativistic limit, $T \gg \mu$, on the other hand

$$
\begin{gathered}
w \stackrel{T \gg \mu}{\longrightarrow} T s, \\
v \stackrel{T \gg \mu}{\longrightarrow} \frac{\eta}{T s}
\end{gathered}
$$

Thus, in a sense the ratio $\eta / s$ is measure of the kinematic viscosity for relativistic systems with small chemical potential but certainly not for systems like water, where the enthalpy-density is dominated by the mass-density.

To define a more precise measure of fluidity, let us analyze the propagation of sound modes. In the linearized regime, the dispersion relation for a sound mode is given by

$$
\omega=c_{s} k-\frac{i}{2} k^{2} \times\left\{\begin{array}{lr}
\frac{\frac{4}{3} \eta}{w / c^{2}}, & \text { R fluid } \\
\frac{4}{3} \eta & \text { NR fluid }
\end{array}\right\}
$$

for a relativistic (R) fluid and non-relativistic (NR) fluid, respectively. We note in passing, that the imaginary part, i.e. the dissipation, is proportional to the square of the wave-number $k$ which reflects the fact that fluid-dynamics works always in the long wavelength limit. By requiring that the ratio of the imaginary over the real part of the sound frequency is small, we ensure that the sound mode propagates well. Or in other word the condition

$$
\left|\frac{I m \omega}{\operatorname{Re} \omega}\right| \ll 1
$$

results in a length-scale $L_{\eta}$

$$
L_{\eta} \equiv\left\{\begin{array}{ll}
\frac{\eta}{\left(w / c^{2}\right) c_{s}}, & \text { R fluid } \\
\frac{\eta}{\rho c_{s}}, & \text { NR fluid }
\end{array}\right\}
$$

which measures the minimum wavelength for which sound propagates with little damping while for the shorter wavelengths dissipation becomes important. For a more precise discussion we refer to [8]. As shown in [8], in the limit of dilute gases, this length-scale $L_{\eta}$ is directly related to the mean free path, $L_{\eta} \sim \lambda_{m f}$, which can be defined for such a system. However, $L_{\eta}$ is not restricted to dilute systems and can be extracted for any system, as it is defined in terms of physical quantities, which do not required special conditions such as a dilute gas.

The final step is to relate $L_{\eta}$ to a length-scale intrinsic to the system. This is necessary in order to be able to compare system at vastly different length scales such as water, interstellar dust and the QGP. The most obvious scale is the inter-particle distance $d=n^{1 / 3}$, which is well defined for non-relativistic systems. For relativistic systems made out of quasi-particles one can extract the inter-particle distance from the entropy density $d \sim s^{1 / 3}$. If no quasi-particle picture applies the typical length-scale of a energy-density correlation function may serve the purpose, which would also apply for other systems [8].

Thus we define the fluidity measure $\mathcal{F}$ as

$$
\mathcal{F} \equiv \frac{L_{\eta}}{L_{n}}
$$

with

$$
L_{n}=\frac{1}{n^{\frac{1}{3}}}
$$

The resulting values for $\mathcal{F}$ for a large variety of substances is shown in Fig.1.

Although the critical pressure and temperature as well as the molar mass of the substances shown in Fig. 1 differ by several orders of magnitude, their fluidity is quite similar, especially just below the critical temperature. Thus, we may conclude that "a good fluid is a good fluid". The spike at $T_{c}$ is due to the fact that the velocity of sound, $c_{s}$, vanishes at the critical point.

Since the fluidity measure seems to work rather well, it will be interesting to see how the QGP compares with well known substances such as water. This is shown in Fig. 2. In order to estimate the fluidity measure $\mathcal{F}$ for the QGP, we have used a parametrization of the viscosity $\eta$ by Hirano and Gyulassy [18]. The enthalpy-density $w=$ $\epsilon+p$ and speed of sound $c_{s}$ are taken from recent lattice results by Karsch et al [19] for $2+1$ flavor QCD with $m_{\pi} \approx 220 \mathrm{MeV}$. As we mentioned before, $L_{n}$ is estimated by $1 /\left(s / 4 k_{B}\right)^{1 / 3}$ with the entropy density also taken from 


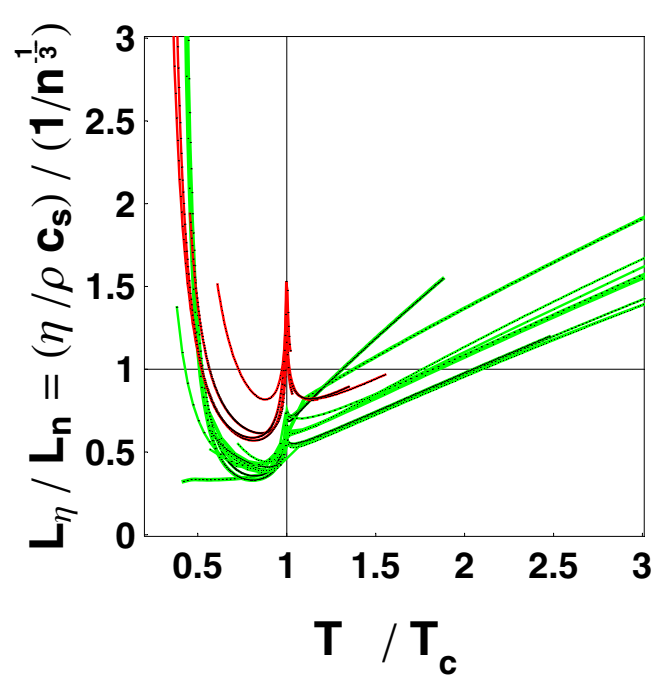

Fig. 1. Fluidity measure $\mathcal{F}=L_{\eta} / L_{n}$ versus $T / T_{c}$ for fifteen different substances $\left(\mathrm{H}_{2},{ }^{4} \mathrm{He}, \mathrm{H}_{2} \mathrm{O}, \mathrm{D}_{2} \mathrm{O}, \mathrm{Ne}, \mathrm{N}_{2}, \mathrm{O}_{2}, \mathrm{Ar}, \mathrm{CO}_{2}\right.$, $\mathrm{Kr}, \mathrm{Xe}, \mathrm{C}_{4} \mathrm{H}_{10}, \mathrm{C}_{8} \mathrm{H}_{18}, \mathrm{C}_{12} \mathrm{H}_{26}, \mathrm{C}_{4} \mathrm{~F}_{8}$ ) at fixed critical pressure $P=P_{c}$. The data are from [17]. The sharp peaks centered at $T_{c}$ are due to the fact that the speed of sound vanishes at $T_{c}$.

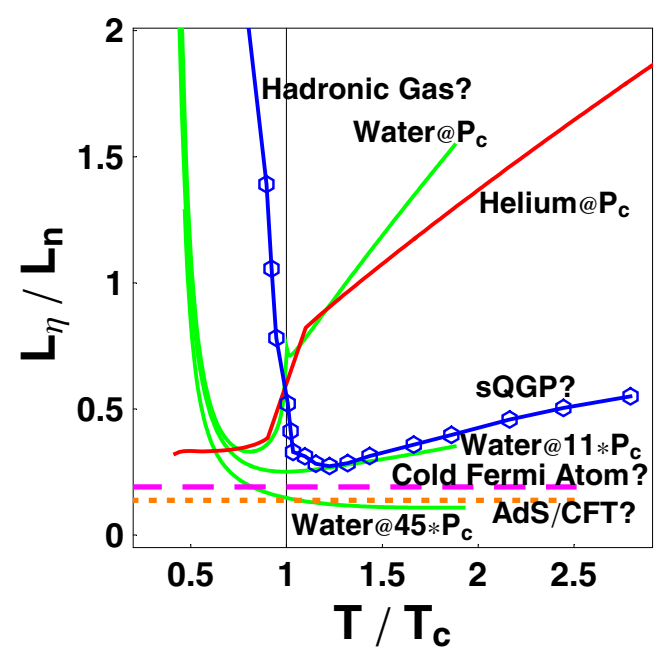

Fig. 2. Comparison of the fluidity measure for various fluids (see text for more details). The curves with question marks indicate current estimates of the respective fluidity with possible uncertainty, while the curves for Helium at $P_{c}$ and for water at $P_{c}, 11 P_{c}, 45 P_{c}$ are from actual data.

[19]. For the strongly coupled AdS/CFT system, the shear viscosity is well known to be $\eta / s=1 /(4 \pi)$ [4]. As we also pointed out before, there is a short-range order at the length scale $L_{n} \sim 1 / T$ however the pre-factor is not accurately determined. We simply use $L_{n}=1 / T$ as an estimate. This gives the fluidity $\mathcal{F}=\sqrt{3} /(4 \pi) \approx 0.138$. For the Cold Fermi Atom gas we used available measurements for its shear viscosity [20] and the speed of sound [21]. For details see [8]. Finally we also plot the fluidity measure for supercritical water at $P=11 P_{C}$ and $P=45 P_{c}$. Surprisingly we find that the fluidity of super-critical water is better or at least comparable with both the QGP and the strongly coupled AdS/CFT system. Super-critical fluids are substances

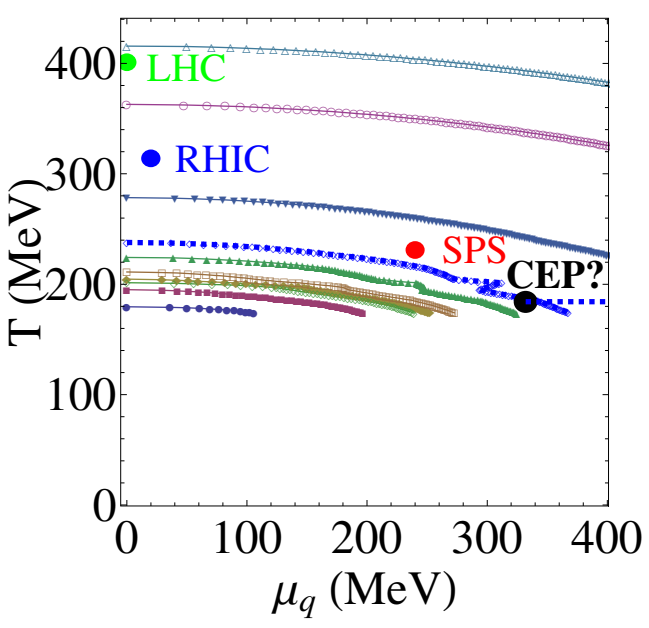

Fig. 3. Schematic isobaric contours (i.e. with constant pressure along each line) on the QCD $T-\mu$ phase diagram with the filled black circle indicating a possible position of the hypothetical Critical-End-Point (CEP); the dashed blue horizontal short line stretching from the CEP to the right is included to indicate the $T=T_{C E P}$ boundary (see text for more details). The filled red, blue and green circles indicate estimates of the initial $(T, \mu)$ reachable at SPS, RHIC and LHC, respectively.

with both the pressure and the temperature above the critical point. They have the interesting property that they are compressible but at the same time behave like fluids, as can be seen from our plot. Since it is expected that the QCD phase-diagram exhibits a critical point [22], it is natural to speculate if the QGP may be a super-critical fluid as well. This may very well be the case, as demonstrated in Fig. 3, where we show the estimated starting point for RHIC and LHC fluid-dynamic evolutions together with equal pressure line in the QCD phase diagram. We also indicate a possible position of the QCD critical point, noting that its existence and precise location is still very much unknown. What would be the consequences if indeed the QGP were a super-critical fluid. Obviously, the system created at the LHC would then sit at even higher temperature and pressure and thus should exhibit an even better fluidity. And since the system at the LHC lives considerable longer one would predict an even better description of the expansion dynamics in terms of fluid-dynamics. To which extent this is borne out by the recent measurement of elliptic flow at the LHC is questionable. The dependence of $v_{2}$ on the transverse momentum is essentially the same as observed for RHIC collisions, whereas a better fluidity would predict a behavior more similar to ideal fluid-dynamics. However, before one can draw any firm conclusions, the effect of jet-like correlations on the elliptic flow measurements at higher transverse momentum needs to be understood [23].

Let us conclude this section with a few general remarks. First, the ratio $\eta / s$ is not suitable to compare the fluidity of different substances. It may serve as a measure for relativistic fluids far away from a phase-transition, where the speed of sound is essentially a constant. Second, while $\eta / s$ may be small for a Quark Gluon Plasma, this does not mean that the sheer viscosity itself is small. Indeed, using 
the conjectured lower limit of $\eta / s=1 /(4 \pi)$ and inserting the value for the entropy density of a QGP one arrives at

$$
\eta_{Q G P} \simeq 2 \times 10^{9} \mathrm{~Pa} \mathrm{~s} \simeq 10^{12} \eta_{\text {Water }}
$$

which is probably the most "sticky" substance known. In comparison, tar-pitch is estimated to have $\eta_{\text {tar }} \simeq 10^{8} \mathrm{~Pa} \mathrm{~s}$. Thus the QGP flows only so well because it has such a large inertia. The large value of the shear-viscosity itself could be exposed if one would be able to drag a small object through the QGP. In this case Stokes' formula for the force required to move an object of radius $R$ at constant speed $u$ is

$$
F=6 \pi R u \eta
$$

Even if we rescaled the size of the sphere by the interparticle distance, $R_{Q G P} \simeq 10^{-6} R_{\text {Water }}$, the force required to drag this sphere through the QGP would still be six orders of magnitude larger than that for water

$$
F_{Q G P}\left(R_{Q G P}\right) \simeq 10^{6} F_{\text {Water }}\left(R_{\text {Water }}\right)
$$

A sticky substance indeed! Expressing this drag force in terms of more conventional units,

$$
F_{Q G P} \simeq 5 R u \frac{\mathrm{GeV}}{\mathrm{fm}^{2}}
$$

so that a particle with radius of $R=0.5 \mathrm{fm}$ and a velocity of $u=0.5$ would feel a drag force or energy loss of $F=d E / d x \simeq 1.25 \mathrm{GeV} / \mathrm{fm}$. To which extent this observation has any bearing on the observed energy loss of heavy particle, remains to be seen.

\section{Local parity violation in heavy ion collisions?}

As briefly discussed in the introduction, the Chiral Magnetic Effect (CME) leads to the separation of charges along the direction of the magnetic field generated by the moving ions. This charge separation can be viewed as a dipole in momentum space as depicted in Fig. 4. In case of the CME, in a given event the dipole vector will be either parallel or anti-parallel to the magnetic field, depending on the presence of sphaleron- or anti-sphaleron transitions in the reaction. Therefore, the expectation value of the momentumspace dipole-moment vanishes, $\langle\mathbf{d}\rangle=0$, as does the expectation value of the parity-odd scalar product with the magnetic field, $\langle\mathbf{B d}\rangle=0$. However, since in case of charge separation $\left\langle\mathbf{d}^{2}\right\rangle \neq 0$ the presence of an event-by-event electric dipole may be observable in the variance of a parity-odd operator, or equivalently, in charge-dependent two-particle correlations. Of course, simple statistical fluctuations also give rise to a finite $\left\langle\mathbf{d}^{2}\right\rangle$ and suitable observables have to be devised which are not sensitive to these statistical fluctuations (for a discussion see [14]).

One way to obtain information about the presences of the CME is to study charge dependent two-particle correlations with respect to the reaction plane, as proposed

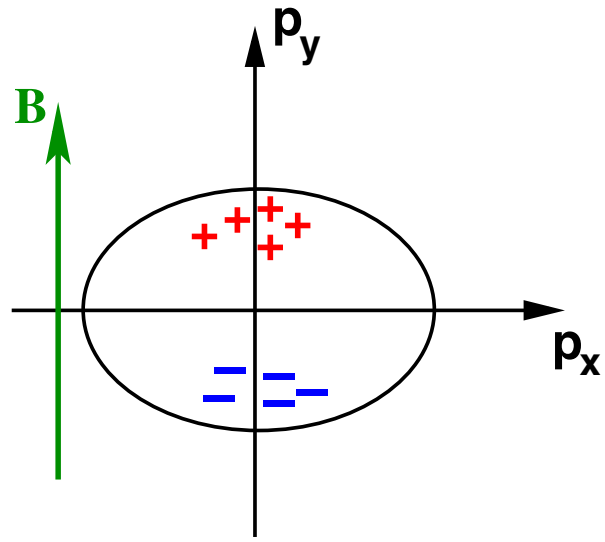

Fig. 4. A schematic illustration of charge separation due to the Chiral Magnetic Effect in an heavy ion event. The reaction plane is aligned along the $p_{x}$-direction on this case.

by Voloshin [10]. He suggested to measure the following three-particle correlation,

$$
\left\langle\cos \left(\phi_{i}+\phi_{j}-2 \phi_{k}\right)\right\rangle
$$

for same-charge pairs $(i, j=++/--)$ and opposite-charge pairs $(i, j=+-)$ with the third particle, denoted by index $k$, having any charge. If the correlation with the third particle $k$ is dominated by elliptic flow, then

$$
\left\langle\cos \left(\phi_{i}+\phi_{j}-2 \phi_{k}\right)\right\rangle=v_{2}\left\langle\cos \left(\phi_{i}+\phi_{j}-2 \Psi_{R . P .}\right)\right\rangle
$$

where $\Psi_{R . P .}$ is the angle of the reaction plane, and $v_{2}$ denotes the strength of the elliptic flow. Working in a frame where the reaction plane is along the x-axis, $\Psi_{R . P .}=0$, we get

$$
\gamma \equiv \frac{1}{v_{2}}\left\langle\cos \left(\phi_{i}+\phi_{j}-2 \phi_{k}\right)\right\rangle=\left\langle\cos \left(\phi_{i}+\phi_{j}\right)\right\rangle
$$

The STAR collaboration has recently measured this correlator and indeed has verified the above dependence of the elliptic flow. Before we discuss the STAR measurement in detail, however, let us see what to expect for this observable in case of the CME. As can be seen from Fig. 4, the CME predicts same-side out-of-plane correlations for same charges and back-to-back out-of-plane correlations for opposite charges. This is best seen by rewriting the correlator $\gamma$ as

$$
\begin{aligned}
\gamma=\left\langle\cos \left(\phi_{i}+\phi_{j}\right)\right\rangle= & \left\langle\cos \left(\phi_{i}\right) \cos \left(\phi_{j}\right)\right\rangle \\
& -\left\langle\sin \left(\phi_{i}\right) \sin \left(\phi_{j}\right)\right\rangle
\end{aligned}
$$

In this representation the first term, $\left\langle\cos \left(\phi_{i}\right) \cos \left(\phi_{j}\right)\right\rangle$, measures the in-plane correlations while the second term, $\left\langle\sin \left(\phi_{i}\right) \sin \left(\phi_{j}\right)\right\rangle$, measures the out-of-plane correlations. The CME predicts that same-charge pairs have either both an angle of $\phi_{i}, \phi_{j} \simeq \pi / 2$ or $\phi_{i}, \phi_{j} \simeq 3 \pi / 2$. In either case, $\sin \left(\phi_{i}\right) \sin \left(\phi_{j}\right) \simeq 1$. For opposite charges, $\phi_{i} \simeq \pi / 2 ; \phi_{j} \simeq$ $3 \pi / 2$ or vice versa and $\sin \left(\phi_{i}\right) \sin \left(\phi_{j}\right) \simeq-1$. Hence the CME predicts

$$
\begin{array}{r}
\gamma_{C M E, \text { same-charge }}<0 \\
\gamma_{C M E, \text { opposite-charge }}>0,
\end{array}
$$




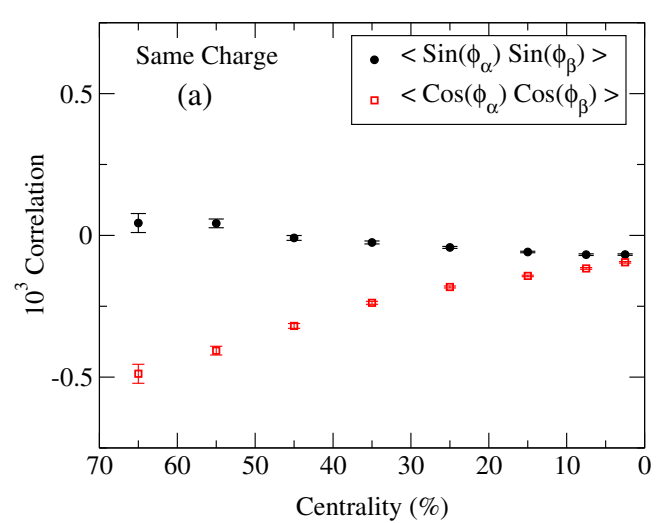

Fig. 5. In-plane (red) and out-of-plane (black) correlations for same-charge pairs as measured by the STAR collaboration [11, 12]. For details see [13].

and indeed this is what the STAR measurement shows. So have we seen the CME and thus local parity violation in an actual experiment? Not quite yet, because there is an alternative scenario for which the correlator $\gamma$ may be negative for same charge pairs and positive for opposite charge pairs: Suppose we have same-charge in-plane backback correlations, i.e. $\phi_{i} \simeq 0$ and $\phi_{j} \simeq \pi$ or vice verse, and opposite-charge in-plane same-side correlations, i.e. $\phi_{i}, \phi_{j} \simeq 0$ or $\phi_{i}, \phi_{j} \simeq \pi$ we obtain the same signs for $\gamma$ as above, but this time it is the $\left\langle\cos \left(\phi_{i}\right) \cos \left(\phi_{j}\right)\right\rangle$ term which controls things. In other words, the correlator $\gamma$ is not unique and we need another observable to determine whether we are dealing with in-plane or out-of-plane correlations. The obvious candidate is

$$
\begin{aligned}
\delta \equiv\left\langle\cos \left(\phi_{i}-\phi_{j}\right)\right\rangle= & \left\langle\cos \left(\phi_{i}\right) \cos \left(\phi_{j}\right)\right\rangle \\
& +\left\langle\sin \left(\phi_{i}\right) \sin \left(\phi_{j}\right)\right\rangle
\end{aligned}
$$

which represents the sum of the in-plane $\left(\left\langle\cos \left(\phi_{i}\right) \cos \left(\phi_{j}\right)\right\rangle\right)$ and out-of plane $\left(\left\langle\sin \left(\phi_{i}\right) \sin \left(\phi_{j}\right)\right\rangle\right)$ correlations. With both $\gamma$ and $\delta$ we can extract both in-plane and out-of-plane correlations separately

$$
\begin{aligned}
\left\langle\cos \left(\phi_{i}\right) \cos \left(\phi_{j}\right)\right\rangle & =\frac{1}{2}(\delta+\gamma)(\text { in }- \text { plane }) \\
\left\langle\sin \left(\phi_{i}\right) \sin \left(\phi_{j}\right)\right\rangle & =\frac{1}{2}(\delta-\gamma)(\text { out }- \text { of }- \text { plane })
\end{aligned}
$$

Fortunately, STAR has measured the correlator $\delta$ allowing for a decomposition of the in-plane and out-of-plane correlations. Those are shown in Fig. 5 for same-charge pairs and in Fig. 6 for opposite charge pairs. The surprising result is that a) for same charge pairs the measured out of-plane correlations are essentially zero, in contrast to the predictions from the CME. Instead STAR observes an inplane back-to-back correlation! This situation is illustrated in Fig. 7. Opposite-charge pairs, on the other hand seem to be equally correlated in both the in-plane and out-ofplane direction. Obviously this is not quite in agreement with the expectation from the CME. Especially the fact that the same-charge pairs do not show any out-of-plane correlations for all centralities is difficult to understand in the context of the CME predictions. Naturally, there will be

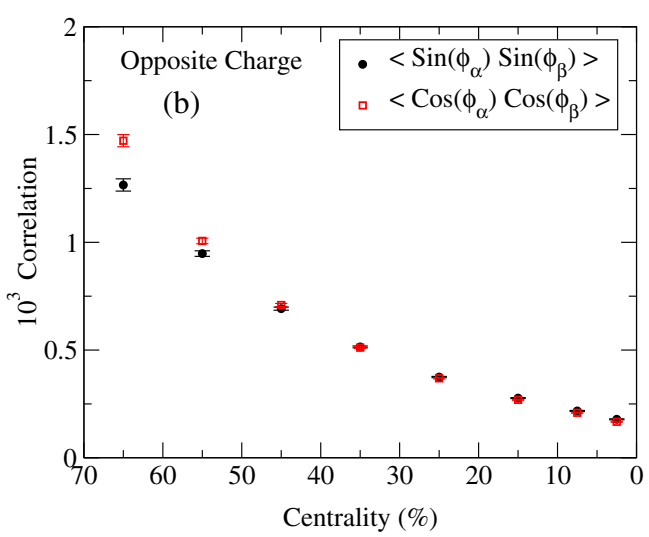

Fig. 6. In-plane (red) and out-of-plane (black) correlations for opposite-charge pairs as measured by the STAR collaboration $[11,12]$. For details see [13].

\section{Chiral Magnetic Effect}

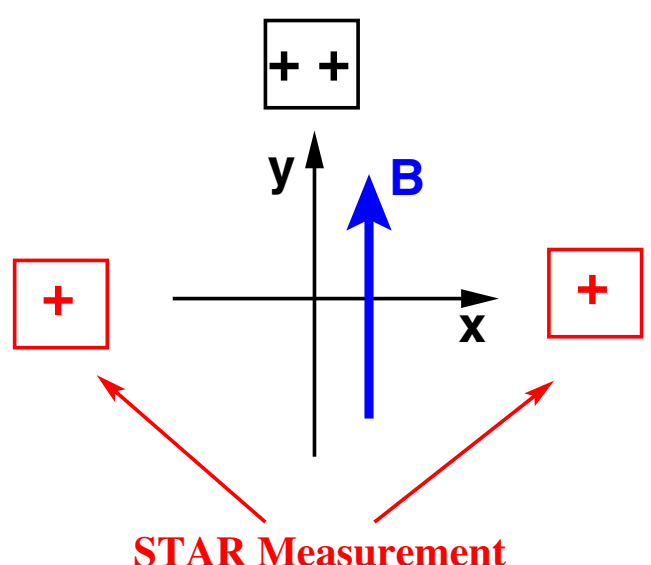

Fig. 7. Schematic illustration of the actual STAR measurement (red) together with the predictions from the Chiral Magnetic Effect (black) for same-charge pairs.

other effects contributing to the correlators $\gamma$ and $\delta$, such as the coulomb interaction, transverse-momentum conservation[15], local charge conservation [24,25], cluster-decays [26] etc. However, it is difficult to imagine how for all centralities these "background" contributions conspire to perfectly cancel the correlations expected from the CME. One should note, however, that so far the measured correlation are not understood in terms of conventional physics either, possibly because many effects contribute, as indicated above. Furthermore, it would be very useful to have a more differential information on the above correlations. While STAR has extracted the rapidity and transverse-momentum dependence of $\gamma$ this information is not yet available for $\delta$. In addition, the value for the above correlations in simple proton-proton collisions would serve as an important reference point. It may also be useful to develop alternative observables [14,27]. For example in [14] the direct extraction of the magnitude and direction with respect to the reaction plane of the momentum-space dipole-moment has been proposed by introducing a charge-dependent Qvector analysis [1]. In [14] it was also demonstrated that 
simple two-particle correlations may mimic the effect of an actual dipole, and only the careful analysis of the distributions of both the magnitude and the angle of the extracted dipole was able to distinguish between an explicit dipole and other correlations.

\section{Conclusions}

In this contribution we have discussed two aspects of the physics of dense matter. On the one hand we presented a universal measure for the fluidity of any substance and we argued that the widely used measure $\eta / s$ is not suitable for a comparison between non-relativistic and relativistic fluids. Given our new measure, the fluidity of the QGP is not any better than that of water. We further pointed out, that super-critical fluids exhibit an exceptionally good fluidity and speculated to which extent the QGP may be considered a super-critical fluid. We also reminded ourselves that the shear viscosity of the QGP is actually extremely large, and that it only flows so well because of its high inertia, i.e. energy density. This large viscosity could be revealed by dragging an object through the QGP and the resulting drag-force turns out to be of the same magnitude as the energy loss extracted from the analysis of leading particle suppression.

In the second part we critically examined the STAR measurement of charge dependent two and three particle correlations and their relevance for local parity violation. We found that for same charge pairs STAR measures inplane back-to-back correlations in contradistinction to the prediction from the Chiral Magnetic effect, which predicts out-of plane same side correlations. Therefore, the jury on the existence of local parity violation in heavy ion collision is still out.

\section{Acknowledgments}

This work was supported by the Director, Office of Science, Office of High Energy and Nuclear Physics, Division of Nuclear Physics, and by the Office of Basic Energy Sciences, Division of Nuclear Sciences, of the U.S. Department of Energy under Contract No. DE-AC03-76SF00098 and DE-AC02-98CH10886 as well as by the Polish Ministry of Science and Higher Education, grant No. N202 125437.

\section{References}

1. S.A. Voloshin, A.M. Poskanzer, R. Snellings, Collective Phenomena in Non-Central Nuclear Collisions Landolt-Boernstein New Series I/23, Editor: R. Stock, Springer (2010), arXiv: 0809. 2949.

2. U.W. Heinz, Early collective expansion: Relativistic hydrodynamics and the transport properties of $Q C D$ matter in Landolt-Boernstein New Series I/23, Editor: R. Stock, Springer (2010) arXiv: 0901 . 4355.
3. D.A. Teaney, Viscous Hydrodynamics and the Quark Gluon Plasma Quark Gluon Plasma 4, Editors: R.C. Hwa and X.N. Wang, World Scientific, Singapore,(2010), arXiv: 0905. 2433.

4. G. Policastro, D. Son, A. Starinets, Phys.Rev.Lett. 87, 081601 (2001), hep-th/0104066.

5. L.P. Csernai, J. Kapusta, L.D. McLerran, Phys.Rev.Lett. 97, 152303 (2006), nucl-th/0604032.

6. R.A. Lacey, N. Ajitanand, J. Alexander, P. Chung, W. Holzmann et al., Phys.Rev.Lett. 98, 092301 (2007), nucl-ex/0609025.

7. T. Schafer, D. Teaney, Rept.Prog.Phys. 72, 126001 (2009), arXiv:0904.3107.

8. J. Liao, V. Koch, Phys. Rev. C81, 014902 (2010), arXiv: 0909. 3105.

9. D.E. Kharzeev, L.D. McLerran, H.J. Warringa, Nucl.Phys. A803, 227 (2008), arXiv:0711.0950.

10. S.A. Voloshin, Phys.Rev. C70, 057901 (2004), hep-ph/0406311.

11. B. Abelev et al. (STAR Collaboration), Phys.Rev.Lett. 103, 251601 (2009), arXiv:0909.1739.

12. B. Abelev et al. (STAR Collaboration), Phys.Rev. C81, 054908 (2010), arXiv: 0909. 1717.

13. A. Bzdak, V. Koch, J. Liao, Phys. Rev. C81, 031901 (2010), arXiv:0912.5050.

14. J. Liao, V. Koch, A. Bzdak, Phys. Rev. C82, 054902 (2010), arXiv: 1005.5380.

15. A. Bzdak, V. Koch, J. Liao Phys. Rev. C83, 014905 (2010), arXiv: 1008. 4919.

16. L. Landau, L. Lifshitz, Hydrodynamics (Pergamon Press, New York, 1980).

17. National Institute of Standards and Technology (NIST) Standard Reference Database No. 69, June 2005, NIST Chemistry WebBook: http://webbook.nist.gov/chemistry/. See, in particular, Thermophysical Properties of Fluid Systems: High Accuracy Data for a Select Group of Fluids.

18. T. Hirano, M. Gyulassy, Nucl.Phys. A769, 71 (2006), nucl-th/0506049.

19. M. Cheng, N. Christ, S. Datta, J. van der Heide, C. Jung et al., Phys.Rev. D77, 014511 (2008), arXiv: 0710.0354 .

20. A. Turlapov, et al, J. Low. Temp. Phys. 150, 567 (2008); T. Schaefer, Phys. Rev. A 76, 063618 (2007).

21. J. Joseph, et al, Phys. Rev. Lett. 98, 170401 (2007).

22. M.A. Stephanov, Prog. Theor. Phys. Suppl. 153, 139 (2004), hep-ph/0402115.

23. J. Liao, V. Koch, Phys. Rev. Lett. 103, 042302 (2009), arXiv: 0902 . 2377.

24. S. Pratt (2010), arXiv: 1002.1758.

25. S. Pratt, S. Schlichting, S. Gavin (2010), arXiv: 1011.6053.

26. F. Wang, Phys.Rev. C81, 064902 (2010), arXiv:0911. 1482.

27. N. Ajitanand, R.A. Lacey, A. Taranenko, J. Alexander (2010), arXiv: 1009. 5624. 